\title{
Inferior Epigastric Artery Pseudoaneurysm Following Paracentesis in a Liver Graft Recipient: A Case Report
}

\author{
Amir Pasha Ebrahimi, ${ }^{1}$ Mohsen Nasiri Toosi, ${ }^{2,3}$ Setareh Davoudi, ${ }^{3,4}$ Ali Jafarian, ${ }^{1,3, *}$ and Hos- \\ sein Ghanaati, ${ }^{3,5,6}$ \\ ${ }^{1}$ Department of Surgery, Hepatobiliary and Liver Transplantation Division, Imam Khomeini Hospital, Tehran University of Medical Sciences, Tehran, Iran \\ ${ }^{2}$ Department of Internal Medicine, Gastroenterology Division, Imam Khomeini Hospital, Tehran University of Medical Sciences, Tehran, Iran \\ ${ }^{3}$ Liver Transplant Research Center, Tehran University of Medical Sciences, Tehran, Iran \\ ${ }_{5}^{4}$ Department of Infectious Diseases, Imam Khomeini Hospital, Tehran University of Medical Sciences, Tehran, Iran \\ 5 Advanced Diagnostic and Interventional Radiology Research Center (ADIR), Tehran University of Medical Sciences, Tehran, Iran \\ ${ }^{6}$ Advanced Diagnostic and Interventional Radiology Research Center (ADIR), Tehran University of Medical Sciences, Tehran, Iran \\ ${ }^{*}$ Corresponding author: Ali Jafarian, Department of Surgery, Hepatobiliary and Liver Transplantation Division, Imam Khomeini Hospital, Tehran University of Medical Sciences, \\ Tehran, Iran. Tel: +98-2166581657, E-mail: jafarian@tums.ac.ir
}

Received: October 21, 2013; Revised: December 15, 2013; Accepted: April 5, 2014

\begin{abstract}
Pseudoaneurysm happens when the artery wall is injured and the blood is contained by the surrounding tissues with eventual formation of a fibrous sac communicating with the artery. We report a case of a 39-year-old man with inferior epigastric artery(IEA) pseudoaneurysm after paracentesis. The pseudoaneurysm was diagnosed by Doppler ultrasound and treated by surgical intervention regarding the patient's underlying comorbidity. IEA false aneurysm must be included in the differential diagnosis during investigation of the cause of any swelling after paracentesis. Cirrhotic patients may be more prone to this complication because of thin rectus muscle that could not confine the hematoma.
\end{abstract}

Keywords: Diagnosis; Epigastric Arteries; Paracentesis; Pseudoaneurysm; Cirrhosis

\section{Introduction}

Pseudoaneurysm happens when the artery wall is injured and the blood is contained by the surrounding tissues with eventual formation of a fibrous sac communicating with the artery. Not many case reports have been published about inferior epigastricartery (IEA) pseudoaneurysm followed by iatrogenic causes including paracentesis (1). We report a case of a 39-year-old man with IEA pseudoaneurysm after paracentesis, which was diagnosed by Doppler ultrasound and treated by surgical intervention regarding to his underlying comorbidity.

\section{Case Presentation}

A 39-year-old man with a history of cirrhosis caused by HCV, who underwent liver transplantation 2 months ago, presented with abdominal pain and fever. Diagnostic paracentesis was performed based on clinically overt ascites. The patient was treated with antibiotics as spontaneous bacterial peritonitis (SBP) was confirmed. Because he did not respond to medical therapy, diagnostic laparoscopy and appendectomy was done for him in the next step. His symptoms did not improve and he had alternating fever. Blood and urine culture was also negative on several exams and there were no sign of pneumonia on his chest images. We decided to repeat paracentesis to confirm the control of SBP.

Paracentesis was done carefully on the site marked by ultrasound with a 16-gauge needle and semi-clear fluid was aspirated. After about 30 minutes, the patient complained of a sudden acute severe pain in his right lower quadrant (RLQ) that was triggered by coughing. In his physical examination, there was a small bulge in the RLQ, which was tender, and no thrill or bruit was detected. The patient's hemodynamic status was stable so he was observed. Few hours later, the bulging expanded following coughs, so an abdominal ultrasound was requested to focus on the RLQ area. An abdominal wall hematoma posterior to rectus muscles was reported with no sign of herniation or arterial flow. The patient's coagulation profile was normal except for INR of 1.5. The bulging did not expand during the next hours, he was hemodynamically stable, and the coagulation tests were normal; therefore, we only corrected the already low hemoglobin level by transfusing packed red blood cells (RBC) and the patient was observed.

Two days later, there was another episode of sudden pain and expanding of the abdominal wall bulging. Urgent abdominal wall Doppler ultrasound reported a massive hematoma posterior to rectus muscles and an IEA pseudoaneurysm (Figure 1). Doppler studies showed a $3 \mathrm{~cm} \times 2 \mathrm{~cm} \times$ $2 \mathrm{~cm}$ oval anechoic mass through the posterior part of the right rectus abdominis muscle. Moreover, IEA pseudoaneurysm was confirmed which was $3 \mathrm{~cm} \times 2.5 \mathrm{~cm} \times 3 \mathrm{~cm}$ in diameter, and consisted of neck $(4 \mathrm{~cm})$ and tract $(9 \mathrm{~cm})$. Also, Doppler studies showed turbulent flow (to and fro sign) within this structure (Figure 2). IEA pseudoaneurysm

Copyright (C) 2015, Tehran University of Medical Sciences and Iranian Society of Radiology. This is an open-access article distributed under the terms of the Creative Commons Attribution-NonCommercial 4.0 International License (http://creativecommons.org/licenses/by-nc/4.0/) which permits copy and redistribute the material just in noncommercial usages, provided the original work is properly cited. 
was confirmed which was $3 \mathrm{~cm} \times 2.5 \mathrm{~cm} \times 3 \mathrm{~cm}$ in diameter, and consisted of neck $(4 \mathrm{~cm})$ and tract $(9 \mathrm{~cm})$.

As the patient had a serum creatinine of $4.4 \mathrm{mg} / \mathrm{dL}$ with a blood urea of $53 \mathrm{mg} / \mathrm{dL}$ that was caused by previously known renal failure, other modalities such as abdominal-pelvic CT scan or MR angiography could not be performed in order to confirm the suggested diagnosis by ultrasound study. Furthermore, therapeutic percutaneous vascular intervention was not possible due to the risk of contrast nephropathy. So, the best diagnostic and therapeutic intervention was operative exploration.

We performed the operation with an oblique incision on the marked site of the false aneurysm by ultrasound and the hematoma was evacuated. IEA false aneurysm was exposed and bleeding from one muscular branch was observed. The hematoma was behind the rectus muscle and it was completely around the anatomic locationof IEA. During operation, for appropriate management of the pseudoaneurysm, we evacuated the hematoma completely, but the IEA bleeding did not stop. Finally, the artery was ligated and sutured. The patient's pain and bulging was resolved following surgery and he was discharged after completion of his antibiotic treatment.

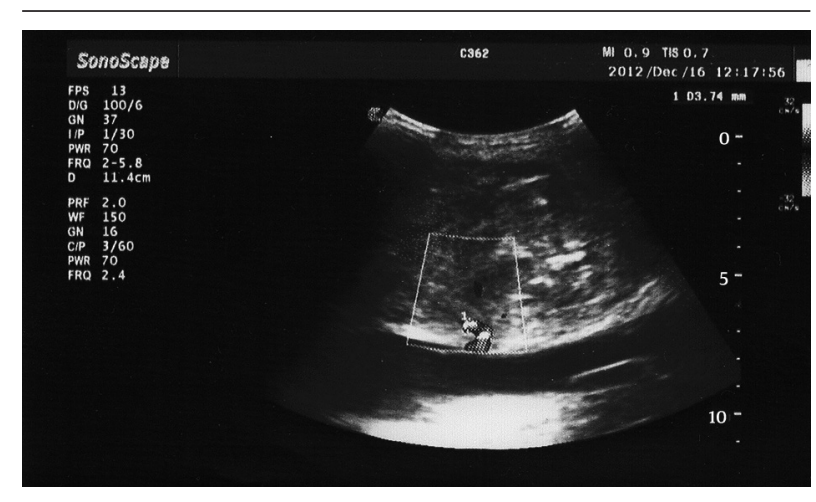

Figure 1. A 39-year-old cirrhotic man with acute severe pain and bulge in the RLQ after diagnostic paracentesis. Doppler study shows IEA pseudoaneurysm.

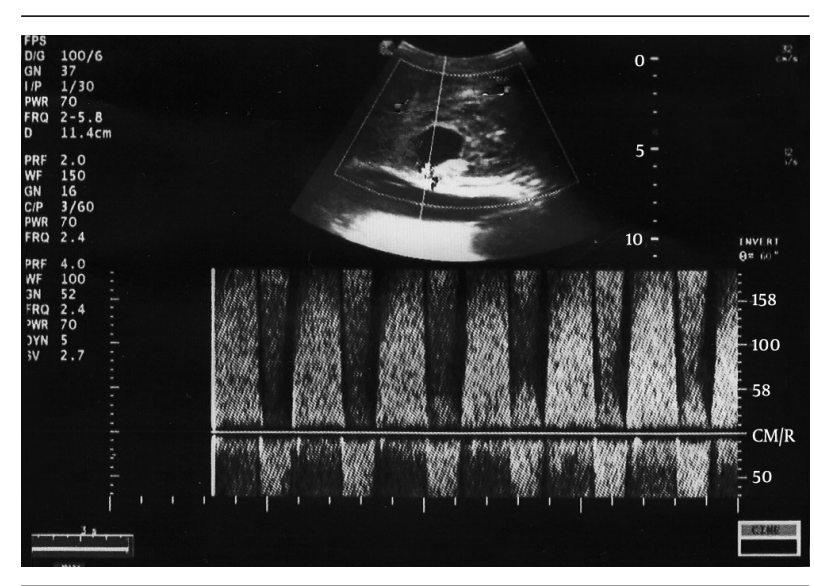

Figure 2. Doppler study shows turbulent flow (to and fro sign) within this structure.
It is noteworthy that by specialist consultations and proper exams, other reasons of extra-abdominal infections were ruled out. Moreover, the patient's abdominal pain was reduced and diagnostic laparoscopy was done to evaluate whether operable reasons exist or not. Also, the patient was immune-suppressed so the sign and symptoms of appendicitis were not reliable. Additionally, the patient did not show the typical manifestation of appendicitis, so there was no indication for appendectomy procedure. While we performed diagnostic laparoscopy, we observed an abnormal appendices and appendectomy was also carried out. We documented it as the patient's past medical history.

\section{Discussion}

IEA pseudoaneurysm is a rare complication that can occur following procedures in the abdomen such as retention sutures, laparoscopy port site, drain insertion, catheter removal, needle biopsy or paracentesis (1-4). In addition, a case was reported following incisional hernia repair (5) as another interesting case of spontaneous pseudoaneurysm (6). The risk of vessel wall injury and following false aneurysm formation is due to its superficial position and its relation to the rectus muscle.

Overall review of the literature showed 20 cases of IEA pseudoaneurysm. Most of them (17 out of 20) showed no bruit and two of them had a pulsatile mass. All of these cases presented a laterally placed abdominal mass (1-8).

In our case, transplantation procedure was done two months before the patient's complications. In the days after transplantation, we did not observe any problems. Moreover, transplantation procedure did not adjust to IEA associated complications, anatomically. The location of laparoscope insertion was far from IEA, and laparoscopy was done several days prior to these events. Therefore, the IEA pseudoaneurysm could not be related to the aforesaid procedures.

IEA pseudoaneurysm is difficult to diagnose clinically. It usually presents as a diffuse, tender mass that is, however, non-pulsatile and also no bruit may be auscultated on it (5). So it can be similar to simple hematomas, especially the ones occurring after procedures including paracentesis that can cause hematoma as a complication. This makes the physical examination less reliable for diagnosing IEA pseudoaneurysm. In our patient, there was no bruit or pulsations in the physical examination and based on the first ultrasound, simple hematoma was thought as the probable diagnosis. This shows the importance of considering IEA pseudoaneurysm as a differential diagnosis in any patient with tender bulging in the abdomen, especially after invasive procedures.

Color Doppler ultrasound is a simple and noninvasive method that helps diagnose pseudoaneurysm. It is the imaging method of choice for evaluation of suspicious masses (8). On ultrasound, hematomas exhibit vari- 
able echogenicity and internal complexity but never demonstrate internal blood flow (1). In contrast, a pseudoaneurysm will be easily demonstrated as an extravasation of blood flow outside the vessel. Characteristic appearances are seen with color Doppler imaging. Typical ultrasonic findings include a focal area of flow with a mixed, swirling red and blue color pattern varying with systole and diastole, a jet between the vessel and the aneurysm sac and the "to and fro" sign on spectral Doppler analysis (9). Review of the literature showed $100 \%$ sensitivity and specificity for Doppler US in differentiating pseudoaneurysms from peri-arterial hematomas (10). Contrast enhanced abdominal CT scan, $\mathrm{CT}$ angiography or conventional angiography are other diagnostic modalities.

Open surgery including suture ligation and excision, endovascular coil embolization or more recently ultrasound guided thrombin injection have been described as possible therapeutic options in the treatment of IEA false aneurysms $(3,7,8,11)$. Ultrasound-guided thrombin injection for false aneurysm has been described as an easy, safe, successful and well tolerated procedure (8). Venkatesh et al. (6) and Shabani and Baxter (9) presented two rare cases of spontaneous IEA pseudoaneurysm. Shabani and Baxter (9) reported a case in which thrombosis in a spontaneous IEA pseudoaneurysm was induced by injection of human thrombin. This technique encompasses US-guided percutaneous injection of $1000 \mathrm{IU}$ of human thrombin trough a 22-gauge spinal needle inserted into the pseudoaneurysmal sac.

Nonsurgical treatment of IEA pseudoaneurysm is not acceptable enough, because it may remain unchanged in size $(1,11)$. US-guided compression is a nonsurgical treatment, but for deep and wide neck pseudoaneurysms it is not sufficient (11). Ferrer et al. (12) stated that surgery is appropriate for large aneurysms and embolization for small ones. Percutaneous trans-catheter approach was suggested by Lam et al. for patients with portal hypertension due to chronic liver failure (1). We chose surgery for this patient because of the contrast usage prohibition and unavailability of thrombin.

This case report has shown that IEA false aneurysm must be included in the differential diagnosis during investigation of the cause of any swelling followed by paracentesis. This would help reduce the chance of misdiagnosis and avoid any inappropriate interventions that may cause increased morbidity. Besides, cirrhotic patients may be more prone to this complication be- cause of the thin rectus muscle that could not confine the hematoma.

\section{Acknowledgements}

The authors would like to appreciate all the hepatobiliary section staff of Imam Khomeini hospital for their help.

\section{Authors' Contributions}

All authors contributed to this project and they fulfilled authorship criteria.

\section{Funding/Support}

There were no funding or support resources for this project.

\section{References}

1. Lam EY, McLafferty RB, Taylor LM, Jr., Moneta GL, Edwards JM, Barton RE, et al. Inferior epigastric artery pseudoaneurysm: a complication of paracentesis. JVasc Surg. 1998;28(3):566-9.

2. Kim JM, Jun NH, Kim HK, Min KT. Pseudoaneurysm in the inferior epigastric artery and scrotal hematoma following removal of a femoral vein catheter -A case report. Korean J Anesthesiol. 2010;59 Suppl:S3-5.

3. Gage TS, Sussman SK, Conard FU, 3rd, Hull D, Bartus SA. Pseudoaneurysm of the inferior epigastric artery: diagnosis and percutaneous treatment. AJR Am J Roentgenol.1990;155(3):529-30.

4. Williamson JS, Harries RL, Davies G, Woodward A. Spontaneous resolution of an inferior epigastric artery pseudoaneurysm secondary to surgical drain placement. Ann R Coll Surg Engl. 2012;94(6):e193-4.

5. Parnaby CN, Nicholls D, Docherty JG. Inferior epigastric artery false aneurysm following incisional hernia repair. Int J Surg Case Rep. 2011;2(7):178-80.

6. Venkatesh SK, Reynolds VB, Raj Sidhu H, Maran PK. Spontaneous inferior epigastric artery pseudoaneurysm. J Clin Ultrasound. 2010;38(5):259-62.

7. Park SW, Choe WH, Lee CH, Lee MW, Kim YJ, Kwon SY, et al. Transcatheter embolization of a pseudoaneurysm of the inferior epigastric artery with $\mathrm{N}$-butyl cyanoacrylate. Br J Radiol. 2008;81(963):e64-7.

8. Krokidis M, Hatzidakis A, Petrakis J, Lagoudis T, Tsetis D. Coil embolization of inferior epigastric artery pseudoaneurysm after percutaneous thrombin injection failure: a case report. Cases $J$. 2009;2:6562.

9. Shabani AG, Baxter GM. Inferior epigastric artery pseudoaneurysm: ultrasound diagnosis and treatment with percutaneous thrombin. BrJ Radiol. 2002;75(896):689-91.

10. Schwartz RA, Kerns DB, Mitchell DG. Color Doppler ultrasound imaging in iatrogenic arterial injuries. Am J Surg. 1991;162(1):4-8.

11. Georgiadis GS, Souftas VD, Papas TT, Lazarides MK, Prassopoulos P. Inferior epigastric artery false aneurysms: review of the literature and case report. EurJVasc Endovasc Surg. 2007;33(2):182-6.

12. Ferrer JV, Soriano P, Zazpe C, Vicente F, Herrera J, Lera JM. Pseudoaneurysm of the inferior epigastric artery. Pathogenesis, diagnosis, and treatment. Arch Surg. 1996;131(1):102-3. 